\title{
Interaction of drive and incentive motivation under deprivation and satiation'
}

\author{
PATRICIA ELSTAD BLACK, COL UMBIA COLLEGE \\ ROGER W. BLACK, UNIVERSITY OF SOUTH CAROLINA
}

Two groups of rats were trained to run a straight alley under food deprivation (D) with reward being 2 or 10 food pellets (K). Subsequently, both groups were tested under relative food satiation. No D by $K$ interaction was observed, but a significant $K$ effect also failed to occur. A progressive reduction in consummatory behavior was associated with a decline in running during satiation which was interpreted as indicating the extinction of $\mathrm{K}$.

Hull (1952) assumed that drive (D) and incentive motivation (K) combined in a multiplicative fashion, while Spence (1956) assumed that the combination was additive. Most of the subsequent experimental evidence has supported Spence's view (Black, 1965), although Seward and his associates have reported that a statistical interaction of $D$ and $K$ did occur when they employed extreme values of these variables (Seward, Shea, \& Elkind, 1958; Seward \& Proctor, 1960; Seward, Shea, \& Davenport, 1960). These Es defined "low K" as no reward and "low $D$ " as essentially satiation.

Black (1965) argued that the results of Seward et al were not inconsistent with, but rather followed directly from, Spence's interpretation of incentive motivation. According to this view, $K$ is a summary term for the motivational aspects of the hypothetical $\mathbf{r g}^{-\mathrm{s}_{\mathrm{g}}}$ mechanism which is presumed to become conditioned to cues in the runway as a result of S's consummatory activity in the goal box. If Ss are trained under conditions of satiation such that they fail to consume the reward placed in the goal box, $\mathbf{r}_{\mathrm{g}} \mathrm{s}_{\mathrm{g}}$ will not become conditioned to runway cues and no more $\mathrm{K}$ will develop for such Ss than for those which encounter an empty goal box ("zero Wg"). For these reasons, Black (1965) concluded that the results of Seward et al did not provide an adequate basis for distinguishing between the views of Hull and Spence regarding $D$ and $K$.

The present study was designed to investigate the combination of $\mathrm{D}$ and $\mathrm{K}$ under conditions of deprivation and relative satiation, with an attempt being made to insure compliance with Spence's (1956) theoretical requirements for the development of $\mathrm{K}$ prior to testing under satiation. Specifically, two groups of rats were trained in a runway under food deprivation and with either "large" or "small" Wg. Subsequently, both groups were shifted to a condition of relative food satiation. The implications of Spence's position for this situation are as follows: the shift from deprivation to satiation should produce an immediate decrement in performance due to the reduction in $\mathrm{D}$; during the deprivation-acquisition phase Ss receiving "large" Wg should perform at a higher level than "small" Wg Ss; there should be no D by $K$ interaction; and, finally, any failure of the $S s$ to eat their reward under satiation should result in the extinction of $K$ and the consequent decline in the strength of the instrumental $R$.

Method

The Ss were 44 female hooded rats, approximately 120 days old at the beginning of training. The apparatus consisted of a 46 in. straight alley painted flat black and equipped with starting and retrace doors and with photocell circuitry which provided a measure of running speed in the second 6 in. of the alley.

Ten days prior to the beginning of training, Ss were placed on a maintenance schedule consisting of $9 \mathrm{~g}$ of lab chow and ad lib water daily. This schedule was continued throughout the "acquisition-deprivation" phase of the experiment, with an appropriate correction being made for the amount of food each $\mathrm{S}$ consumed as reward in the runway.

On Acquisition-Deprivation Days 1 and 2 each S received two reinforced trials in the runway, while on Days 3-14 Ss received four daily trials. Throughout training and subsequent satiation, an intertrial interval of about $10 \mathrm{~min}$ was maintained. For half of the Ss (G2) reinforcement on each trial consisted of two 45 mg Noyes food pellets, while for the remaining Ss (G10) 10 such pellets served as reward. Following these 14 days of Acquisition-Deprivation training, Ss in both groups were given five additional days of "satiation testing" in which they were allowed $60 \mathrm{~min}$ access to lab chow followed by $30 \mathrm{~min}$ access to a wet mash consisting of ground lab chow, condensed milk, and sugar. Immediately thereafter Ss received their four daily running trials under the same conditions that prevailed during acquisition except that a large supply of Noyes pellets was avallable to each $S$ in its carrying cage in the experimental room and the Ss were, of course, presumably food satiated. On these trials $S$ was removed from the goal box as soon as it had consumed its reward or after $20 \mathrm{sec}$, whichever came earlier.

\section{Results and Discussion}

Running speeds for both groups on the final day of acquisition and on the five days of satiation are presented in Fig. 1. An analysis of performance on the last day of acquisition and the first day of satiation indicated an immediate decrement in the performance of both groups following the satiation procedure, $F=216.2$, df $=1 / 42, p<.001$. Theoretically this decrement was expected on the basis of a reduction in generalized 
drive. The interaction of $D$ and $K$ in this analysis proved to be nonsignificant, $F<1.00, \mathrm{df}=1 / 42$. This result was, of course, also in agreement with Spence's position. Unfortunately, interpretation of the nonsignificant $D$ by $K$ interaction is not unequivocal, since the main effect of Wg during acquisition also proved to be nonsignificant, $\mathrm{F}<1.00, \mathrm{df}=1 / 42$. Thus, a reliable difference in $\mathrm{K}$ apparently failed to develop.

An analysis of variance performed on performance on the five days of satiation indicated that Ss in G2 ran more quickly than those in G10 during this period, $F=9.04, d f=1 / 42, p<.005$. This confirmed a previous result to the effect that performance under satiation is inversely related to the Wg employed during acquisition (Guild, 1960). A possible explanation of this phenomenon is suggested in Fig. 2, which depicts the percent of their available $\mathrm{Wg}$ consumed by Ss in G2 and G10. Examination of this figure suggests that Ss in G2 ate more consistently than those in G10 and, hence, the former may have had less opportunity to engage in nonconsummatory behavior in the goal box. According to Spence (1956) the occurrence of such behavior will result in the extinction of $K$. In fact, the Ss in G2 did tend to eat their reward immediately upon entering the goal box and were thus almost immediately removed, while Ss in G10 tended to stay in the goal box for most or all of the $20 \mathrm{sec}$ they were allowed, and during most of this period they were not eating. It should be noted that similar observations have been previously reported (Black \& Elstad, 1964).

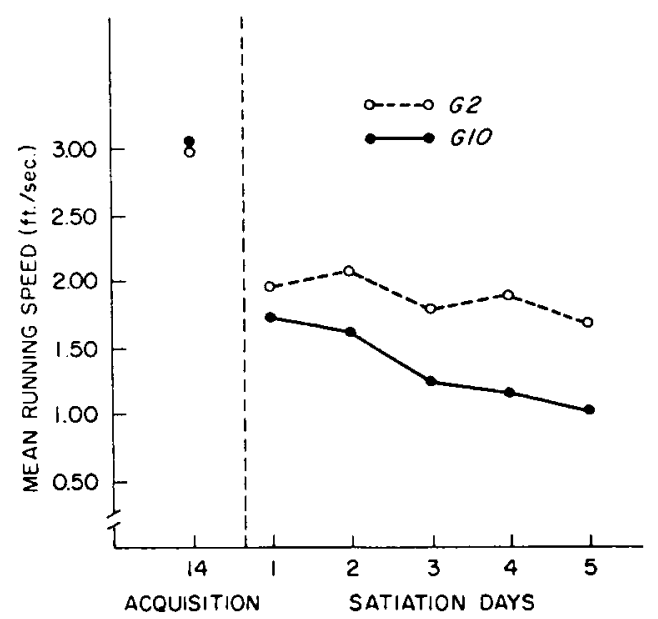

Fig. 1. Mean running speed on the last day of acquisition and the five days of satiation.

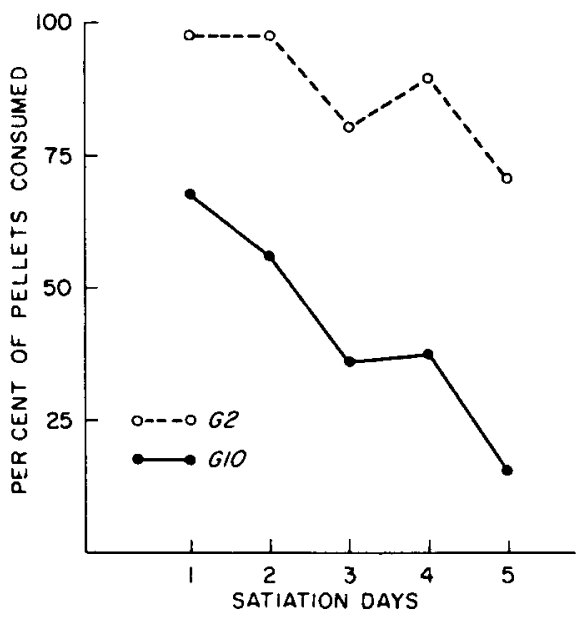

Fig. 2. Mean percent of available pellets consumed by Ss in G2 and $\mathbf{G 1 0}$.

Inspection of both Figs. 1 and 2 indicates that, in spite of the feeding procedure, both instrumental and consummatory Rs showed considerable strength and persistence. Nevertheless, a significant decline in running speed did occur over the five satiation days, $F=12.79$, df $=4 / 160, p<.001$. Presumably the gradual and reliable decline in running speed observed during this satiation period reflected the progressive weakening or extinction of $\mathrm{K}$ occurring during that period.

\section{References}

Black, R. W. On the combination of drive and incentive motivation. Psychol. Rev., 1965, 72, 310-317.

Black, R. W., \& Elstad, Patricia A. Instrumental and consummatory behavior as a function of length of reward period. Psychon. Sci., $1964,1,301-302$.

Guild, R. E. Incentive motivation in satiated rats. J. comp. physiol. Psychol., 1960, 53, 351-358.

Hull, C. L. Principles of behavior. New York: Appleton-CenturyCrofts, 1943.

Seward, J. P., Shea, R. A., \& Elkind, D. Evidence for the interaction of drive and reward. Amer. J. Psychol., 1958, 71, 404-407.

Seward, J. P., Shea, R. A., \& Davenport, R. H. Further evidence for the interaction of drive and reward. Amer. J Psychol., 1960, 23, 370-379.

Seward, J. P., \& Proctor, D. M. Performance as a function of drive, reward and habit strength.' Amer. J. Psychol., 1960, 73, 448-453.

Spence, K. W. Behavior theory and conditioning. New Haven: Yale University Press, 1956.

\section{Note}

1. This report is based on a thesis submitted by the first author to the Graduate College of the University of Iowa in partial fulfillment of the requirements for the degree, Master of Arts. 